\title{
Design, objectives, and lessons from a pilot 25 year follow up re-survey of survivors in the Whitehall study of London Civil Servants
}

Robert Clarke, Elizabeth Breeze, Paul Sherliker, Martin Shipley, Linda Youngman, Astrid Fletcher, Rebecca Fuhrer, Dave Leon, Sarah Parish, Rory Collins, Michael Marmot

Clinical Trial Service Unit and Epidemiological Studies Unit, Nuffield Department of Clinical Medicine, Radcliffe Infirmary, Oxford, OX2 6HE

R Clarke

P Sherliker

L Youngman

S Parish

R Collins

Department of Epidemiology, London School of Hygiene and Tropical Medicine,

London

E Breeze

A Fletcher

D Leon

Department of

Epidemiology and Public Health,

University College

London Medical

School, London

M Shipley

R Fuhrer

M Marmot

Correspondence to: Dr Clarke.

Accepted for publication 23 September 1997

\begin{abstract}
Design-To assess the feasibility of conducting a re-survey of men who are resident in the United Kingdom 25 years after enrollment in the Whitehall study of London Civil Servants.

Methods-A random sample of 401 study survivors resident in three health authority areas was selected for this pilot study. They were mailed a request to complete a self administered questionnaire, and then asked to attend their general practice to have their blood pressure, weight, and height measured and a blood sample collected into a supplied vacutainer, and mailed to a central laboratory. Using a $2 \times$ 2 factorial design, the impact of including additional questions on income and of an informant questionnaire on cognitive function was assessed.
\end{abstract}

Results-Accurate addresses were obtained from the health authorities for $96 \%$ of the sample. Questionnaires were received from $73 \%$ and blood samples from $61 \%$ of the sample. Questions on income had no adverse effect on the response rate, but inclusion of the informant questionnaire did. Between 1970 and 1995 there were substantial changes within men in the mean blood pressure and blood total cholesterol recorded, as reflected by correlation coefficients between 1970 and 1995 values of 0.26 , and 0.30 for systolic and diastolic blood pressure and 0.38 for total cholesterol.

Conclusion-This pilot study demonstrated the feasibility of conducting a re-survey using postal questionnaires and mailed whole blood samples. The magnitude of change in blood pressure and blood total cholesterol concentrations within individuals was greater than anticipated, suggesting that such remeasurements may be required at different intervals in prospective studies to help interpret risks associations properly. These issues will be considered in a re-survey of the remaining survivors of the Whitehall study.

(F Epidemiol Community Health 1998;52:364-369)

Much of our knowledge of the determinants of morbidity and mortality in adult life comes from prospective studies of middle aged people. ${ }^{12}$ Accumulating data indicate that blood pressure and blood cholesterol predict cardiovascular risks in people aged 65 to 74 years. But substantial uncertainty remains about the strength of these associations, ${ }^{34}$ and there is even greater uncertainty among any risk associations among people aged over 75 years. ${ }^{5}$ There is also a paucity of information about the impact of socioeconomic advantages in middle age on disease risk in old age. ${ }^{6}$ Socioeconomic circumstances are difficult to define in old age, because the traditional measures (such as occupation, housing tenure, access to a car) may be less relevant after retirement, but information on income may be more informative.

Risk associations in the elderly based on exposures measured in middle age are likely to be complicated by variability over time, comorbidity and concomitant use of medication, all of which may change exposure levels during the follow up period. Hence, to obtain reliable information on these risk associations, and on changes in exposures over time, prospective studies may need to collect risk factor data in middle age and then again in old age. Variability in measurements of exposures (because of analytic error or within person variation) in prospective studies, cause a systematic underestimation of the strength of association between risk factors and disease, referred to as "regression dilution". ${ }^{1-10}$ This regression dilution effect may be particularly relevant in the long term follow up of prospective studies.

The Whitehall study is a prospective study of 19019 male London Civil Servants aged 40-69 years when first examined between 1967 and $1970 .{ }^{11}{ }^{12}$ About 9000 men have survived since enrollment about 25 years ago. All are aged more than 65 years, with $80 \%$ aged between 70 and 84 years, and a median age of 73 years. In preparation for a full re-survey of these survivors, the present pilot study aimed to: examine the ability to contact Whitehall study participants using information supplied by the health authorities with which they are registered; determine their response rate to postal questionnaires, including the separate impact of additional questions on income and of questions relating to possible cognitive function (using an informant questionnaire); and determine their response rate to a request to attend their general practice for measurement of blood pressure, weight, and height and for blood sampling; and to make a preliminary assessment of the changes in these measurements within individuals during 25 years. 
Table 1 Response to requests for questionnaires and blood samples in the pilot resurvey at 25 years of 401 surviving Whitehall Study participants

\begin{tabular}{|c|c|c|c|c|}
\hline & \multirow[b]{2}{*}{$\begin{array}{l}\text { Total number } \\
\text { of subjects }\end{array}$} & \multirow{2}{*}{$\begin{array}{l}\text { Number who returned a } \\
\text { questionnaire Number } \\
\text { (\% of total) }\end{array}$} & \multicolumn{2}{|c|}{ Number who returned a blood sample } \\
\hline & & & $\begin{array}{l}\text { Number } \\
\text { (\% of total) }\end{array}$ & $\begin{array}{l}\text { (\% of questionnaire } \\
\text { respondents) }\end{array}$ \\
\hline \multicolumn{5}{|l|}{ Area of residence } \\
\hline Oxfordshire & 106 & $92(87)$ & $84(79)$ & (91) \\
\hline Bromley & 147 & $111(76)$ & $97(66)$ & (87) \\
\hline Lambeth & 148 & $90(61)$ & $65(44)$ & (72) \\
\hline \multicolumn{5}{|l|}{ Current age } \\
\hline$<70$ & 63 & $47(75)$ & $40(64)$ & (85) \\
\hline $70-74$ & 122 & $100(82)$ & $88(72)$ & (88) \\
\hline $75-79$ & 110 & $82(75)$ & $71(65)$ & (87) \\
\hline $80-84$ & 66 & $43(65)$ & $36(55)$ & (84) \\
\hline$\geqslant 85$ & 40 & $21(53)$ & $11(28)$ & $(52)$ \\
\hline \multicolumn{5}{|l|}{ Employment grade (1970) } \\
\hline Administrative & 40 & $35(88)$ & $31(78)$ & (89) \\
\hline Prof/Executive & 256 & $205(80)$ & $179(70)$ & (81) \\
\hline British Council and Diplomatic Service & 23 & $17(74)$ & $13(57)$ & (77) \\
\hline Clerical & 47 & $17(36)$ & $12(26)$ & $(72)$ \\
\hline Other & 35 & $19(54)$ & $11(31)$ & (58) \\
\hline \multicolumn{5}{|l|}{ Smoking status (1970) } \\
\hline Never & 94 & $75(80)$ & $68(72)$ & (91) \\
\hline Ex-smoker & 149 & $120(81)$ & $104(70)$ & (87) \\
\hline Pipe/cigar & 14 & $9(64)$ & $8(57)$ & (89) \\
\hline Cigarette smoker & 144 & $89(62)$ & $66(46)$ & (74) \\
\hline \multicolumn{5}{|l|}{ Income questions } \\
\hline No & 201 & $147(73)$ & $119(59)$ & (81) \\
\hline Yes & 200 & $146(73)$ & $127(64)$ & (87) \\
\hline \multicolumn{5}{|l|}{ Cognitive impairment questions } \\
\hline No & 202 & $154(76)$ & $137(68)$ & (89) \\
\hline Yes & 199 & $139(70)$ & $109(55)$ & (79) \\
\hline Overall & 401 & $293(73)$ & $246(61)$ & $(84)$ \\
\hline
\end{tabular}

\section{Methods}

IDENTIFICATION OF PARTICIPANTS

The National Health Service Central Register (NHSCR) was used to identify health authorities in the United Kingdom in which the surviving Whitehall study members are currently registered with a family doctor. A total of 600 were lost to follow up either because of emigration or being unregistered with a family doctor. The remaining 8400 men were found to be registered with an address in 96 health authorities in England and Wales, 141 in Scotland, and 21 in Northern Ireland, with 75\% resident in the 28 health authorities of the south east region. Three health authorities were chosen for this pilot study, representing different socioeconomic areas and a random sample of 401 study participants not known to be dead was selected from those registered with Oxfordshire (106), Bromley (147), and Lambeth, Southwark and Lewisham (148) health authorities. The health authorities were able to provide addresses for the study participants who were registered with a family doctor in their area. These addresses were screened using an address enhancement computer software package to verify the accuracy and

Table 2 Mean (SD) values of selected characteristics in 1970 among those who provided both completed questionnaires and blood samples in 1995 ("compliers") and those who did not ("non-compliers"), and values obtained on re-measurement in 1995

\begin{tabular}{llll}
\hline & 1970 & \\
\cline { 2 - 3 } Year of measurement & $\begin{array}{l}\text { Compliers }{ }^{*} \text { in } \\
1995(n=246)\end{array}$ & $\begin{array}{l}\text { Non-compliers in } \\
1995(n=155)\end{array}$ & $1995(n=246)$ \\
\hline Systolic blood pressure (mm Hg) & $132(19)$ & $133(22)$ & $131(17)$ \\
Diastolic blood pressure (mm Hg) & $82(12)$ & $82(14)$ & $81(12)$ \\
Cholesterol (mmol/1) & $4.6(1.3)$ & $4.5(1.1)$ & $5.1(1.3)$ \\
Height $(\mathrm{m})$ & $1.77(0.06)$ & $1.75(0.06)$ & $1.73(0.07)$ \\
Weight $(\mathrm{kg})$ & $76(10)$ & $74(10)$ & $76(11)$ \\
\hline
\end{tabular}

"Compliers" refers to those subjects in the random sample who both completed a questionnaire and provided a blood sample in 1995, and "non-compliers" refers to the remainder of the sample (some of whom may have completed a questionnaire but not provided blood). completeness of the addresses. Just before mailing questionnaires, vital status was again checked with NHSCR to minimise the risk of causing distress to family members of participants who may have died recently. Ethics committee approval was obtained from the three institutions involved.

QUESTIONNAIRE MAILING

A request for consent to participate and a self administered questionnaire was mailed with a prepaid reply envelope to the random sample after the final check of vital status with NHSCR. If a response was not received within one month, then a second questionnaire was sent together with a reminder letter. If no response was received to the first reminder, then a second reminder letter was sent using "recorded" delivery. Using "recorded" delivery, the postal service returned a signed acknowledgement of receipt of the questionnaire to the study office. No further reminders were sent to participants who did not reply to the second reminder letters. The 16 page questionnaire included questions on medical history, smoking, alcohol consumption, current medications, housing tenure, central heating, car access, living arrangements, social support, activities of daily living, and a self rating of current health.

ASSESSMENT OF INCLUSION OF PARTICULAR QUESTIONS

Half of the participants were randomly allocated to receive three additional questions on their current sources of income, including their current net weekly income band, and half were not. Independently, using a $2 \times 2$ factorial design, half of the participants received an "informant" questionnaire, which they were requested to pass to a close relative or friend for 
completion, and half were not. This questionnaire involved 19 questions about any apparent behavioural changes in the study participants since one year earlier that might reflect a deterioration in the participants's memory or mental state. ${ }^{13}$

COLLECTION OF CLINICAL MEASUREMENTS AND BLOOD SAMPLES

Upon receipt of a completed questionnaire from participants, a blood collection kit and a postcard was sent to them, and they were asked to attend their general practice. Enclosed instructions provided asked the general practitioner or practice nurse to measure the study participant's blood pressure after five minutes in the seated position on two occasions, with a minimum interval of one minute between each reading, and to record these measurements on the postcard provided. Participants who had been randomly allocated to receive an informant questionnaire (see above), were also sent a questionnaire on cognitive function, which they were asked to pass to their general practitioner. This involved the GP conducting the Short Blessed Test $^{14}$ when measuring the participants's blood pressure. All participants had their body weight and height recorded without shoes but while wearing normal clothes. A $10 \mathrm{ml}$ blood sample was then to be collected into the vacutainer (containing EDTA and aprotinin), and sent by first class post in the prepaid transport tube and protective plastic envelope provided (which has been
KEY POINTS

- A re-survey of surviving men 25 years after enrollment in the Whitehall Study of London Civil Servants was feasible using postal questionnaires and mailed whole blood samples.

- Questions on income had no adverse effect on the response rate but an informant questionnaire on cognitive function did.

- The magnitude of change in blood pressure and blood cholesterol within subjects was greater than anticipated.

- Remeasurements are required at varying intervals in prospective studies for appropriate interpretation of risk associations.

approved by the Post Office) to a central laboratory in Oxford.

Blood samples were centrifuged on arrival in the laboratory, and the plasma was inspected for haemolysis. A single aliquot of buffy coat from each participant was stored in liquid nitrogen for possible future DNA extraction and analysis. The plasma was divided into multiple aliquots for each participant, some for immediate analysis of cholesterol and apolipoproteins using a Beckmann auto-analyser, and the remainder for storage in liquid nitrogen. Total cholesterol was measured in these plasma samples by an enzymatic method using a Beckmann autoanalyser. (By contrast,

Table 3 Mean values in 1970 and 1995 for quintiles of 1970 measurements together with the ranges and ratio of the ranges, and the correlation coefficients of the 1970 and 1995 values

\begin{tabular}{|c|c|c|c|c|c|c|}
\hline \multirow[b]{2}{*}{ Quintiles (based on 1970 values) } & \multicolumn{3}{|c|}{ Systolic blood pressure $(\mathrm{mm} \mathrm{Hg})$} & \multicolumn{3}{|c|}{ Diastolic blood pressure (mm Hg) } \\
\hline & Subjects (n) & 1970 & 1995 & Subjects $(n)$ & 1970 & 1995 \\
\hline I & 63 & 112 & 142 & 65 & 68 & 78 \\
\hline II & 63 & 124 & 146 & 55 & 77 & 82 \\
\hline III & 46 & 134 & 152 & 51 & 83 & 83 \\
\hline IV & 38 & 145 & 149 & 37 & 90 & 82 \\
\hline V & 27 & 164 & 157 & 27 & 103 & 88 \\
\hline Range V-I & 237 & 52 & 15 & 235 & 35 & 10 \\
\hline \multicolumn{7}{|l|}{ Comparison of 1970: 1995} \\
\hline Ratio of quintile ranges & & 3.5 & & & 3.5 & \\
\hline \multirow[t]{2}{*}{ Inverse of correlation coefficient } & & 3.8 & & & 3.8 & \\
\hline & \multicolumn{3}{|c|}{ Cholesterol (mmol/l) } & \multicolumn{3}{|l|}{ Height (m) } \\
\hline Quintiles (based on 1970 values) & Subjects (n) & 1970 & 1995 & Subjects (n) & 1970 & 1995 \\
\hline I & 55 & 3.54 & 5.28 & 50 & 1.68 & 1.65 \\
\hline II & 46 & 4.44 & 5.35 & 53 & 1.74 & 1.71 \\
\hline III & 38 & 4.99 & 5.90 & 49 & 1.77 & 1.74 \\
\hline IV & 57 & 5.66 & 5.98 & 44 & 1.80 & 1.76 \\
\hline V & 43 & 6.96 & 6.25 & 47 & 1.86 & 1.82 \\
\hline Range V-I & 239 & 3.42 & 0.97 & 243 & 0.18 & 0.17 \\
\hline \multicolumn{7}{|l|}{ Comparison of 1970: 1995} \\
\hline Ratio of quintile ranges & & 3.5 & & & 1.1 & \\
\hline \multirow[t]{2}{*}{ Inverse of correlation coefficient } & & 2.6 & & & 1.2 & \\
\hline & \multicolumn{3}{|l|}{ Weight (kg) } & \multicolumn{3}{|l|}{$B M I\left(k g / m^{2}\right)$} \\
\hline Quintiles (based on 1970 values) & Subjects (n) & 1970 & 1995 & Subjects (n) & 1970 & 1995 \\
\hline I & 47 & 62.51 & 64.36 & 57 & 21.01 & 22.70 \\
\hline II & 47 & 70.94 & 71.19 & 51 & 23.28 & 24.13 \\
\hline III & 53 & 75.93 & 75.47 & 44 & 24.72 & 25.09 \\
\hline IV & 45 & 80.57 & 78.64 & 47 & 26.17 & 26.71 \\
\hline $\mathrm{V}$ & 50 & 90.08 & 87.70 & 40 & 28.89 & 28.42 \\
\hline Range V-I & 242 & 27.57 & 23.34 & 239 & 7.88 & 5.72 \\
\hline \multicolumn{7}{|l|}{ Comparison of 1970: 1995} \\
\hline Ratio of quintile ranges & & 1.2 & & & 1.4 & \\
\hline Inverse of correlation coefficient & & 1.3 & & & 1.6 & \\
\hline
\end{tabular}


Table 4 Mean values in 1970 and 1995 for quintiles of 1970 measurements together with the ranges and ratio of the ranges and the correlation coefficient of the 1970 and 1995 values, before and after exclusion of those with prior disease or medication that may change blood pressure or blood cholesterol values

\begin{tabular}{|c|c|c|c|c|c|c|}
\hline \multirow[b]{3}{*}{ Quintiles (based on 1970 values) } & \multicolumn{6}{|c|}{ Systolic blood pressure $(\mathrm{mm} \mathrm{Hg})$} \\
\hline & \multicolumn{3}{|c|}{ After exclusion of prior disease } & \multicolumn{3}{|c|}{$\begin{array}{l}\text { After exclusion of those receiving blood pressure } \\
\text { lowering medication }\end{array}$} \\
\hline & Subjects (n) & 1970 & 1995 & Subjects (n) & 1970 & 1995 \\
\hline I & 52 & 112 & 142 & 57 & 111 & 141 \\
\hline II & 48 & 124 & 146 & 45 & 124 & 146 \\
\hline III & 33 & 135 & 150 & 35 & 134 & 154 \\
\hline IV & 30 & 145 & 149 & 25 & 145 & 152 \\
\hline V & 21 & 163 & 161 & 14 & 164 & 163 \\
\hline Range V-I & & 51 & 19 & & 53 & 22 \\
\hline \multicolumn{7}{|l|}{ Comparison of 1970: 1995} \\
\hline Ratio of quintile ranges & & & 2.7 & & & 2.4 \\
\hline \multirow[t]{3}{*}{ Inverse of correlation coefficient } & & & 3.1 & & & 3.0 \\
\hline & \multicolumn{6}{|c|}{ Total cholesterol ( $\mathrm{mmol} / \mathrm{l})$} \\
\hline & \multicolumn{3}{|c|}{ After exclusion of prior disease } & \multicolumn{3}{|c|}{$\begin{array}{l}\text { After exclusion of those receiving lipid lowering } \\
\text { medication }\end{array}$} \\
\hline Quintiles (based on 1970 values & Subjects (n) & 1970 & 1995 & Subjects (n) & 1970 & 1995 \\
\hline I & 39 & 3.54 & 5.25 & 47 & 3.54 & 5.30 \\
\hline II & 37 & 4.45 & 5.50 & 36 & 4.43 & 5.27 \\
\hline III & 34 & 4.99 & 5.91 & 31 & 5.00 & 5.91 \\
\hline IV & 40 & 5.67 & 6.19 & 44 & 5.66 & 6.05 \\
\hline V & 30 & 6.78 & 6.49 & 39 & 6.96 & 6.20 \\
\hline Range V-I & & 3.24 & 1.24 & & 3.42 & 0.9 \\
\hline \multicolumn{7}{|l|}{ Comparison of 1970: 1995} \\
\hline Ratio of quintile ranges & & & 2.6 & & & 3.8 \\
\hline Inverse of correlation coefficient & & & 2.3 & & & 2.6 \\
\hline
\end{tabular}

total cholesterol had been measured in 1970 by a Technicon method using capillary blood samples collected from an earlobe with a fine bore tube; as a result, the true values at baseline were expected to have been underestimated by about $10 \% .^{15}{ }^{16}$ )

STATISTICAL METHODS

The response rates for receipt of questionnaires and blood samples were examined by area of residence, age, employment grade, smoking status, and whether or not they received additional questions on income or on cognitive impairment. To assess the magnitude of "regression dilution" over the 25 year period since the baseline survey, respondents were classified into quintiles according to their 1970 exposure levels and the mean values of these quintiles were then compared with the mean values among these same individuals at resurvey in $1995 .{ }^{10}$ The ratio of the difference between the mean values of the highest and lowest quintile groups in 1970 and in 1995 provides an estimate of regression dilution during 25 years. So too does the inverse of the correlation coefficient between values in 1970 and 1995. Estimates of regression dilution were made before and after exclusion of those with concomitant illness (heart attack, stroke, angina or any type of cancer other than skin cancer) or those using any medication that would lower blood pressure or blood cholesterol values. $^{10}$

\section{Results}

RESPONSE TO QUESTIONNAIRE

Addresses were obtained for all 401 study participants in the random sample selected from the three health authorities included in this pilot study. Based on the response rate to ques- tionnaires and the use of "recorded" delivery for the second reminder letters to nonrespondents, reasonably complete and accurate addresses were received from $96 \%$ of the 401 men in the sample. Completed questionnaires were received from 293 (73\% of the random sample, table 1). While questionnaires were received from $55 \%$ of the participants after the initial mailing, they were received after the first and second reminder letters from a further $12 \%$ and $6 \%$, respectively. The response rate varied in the three health authorities, ranging from $87 \%$ in Oxfordshire, to $61 \%$ in Lambeth, Southwark and Lewisham, which may reflect socioeconomic differences, and it declined steeply with increasing age. It was higher in those from the higher socioeconomic groups (as reflected by employment grade in 1970), ranging from $88 \%$ in the administrative grade, to only $36 \%$ in the clerical grades, and was lower in men who were current smokers in 1970. The inclusion of additional questions on income did not seem to affect the questionnaire response rate adversely, but the inclusion of questions on cognitive function did.

\section{RESPONSE TO BLOOD SAMPLE REQUEST}

Postal blood samples were received from $61 \%$ of the pilot study population and from $84 \%$ of the questionnaire respondents (table 1). Differences in response rates for receipt of blood samples were similar to those for questionnaires, even when percentages of those who completed questionnaires are considered. Inclusion of questions on cognitive function seemed to further reduce the response rate of participants. Seventy seven per cent of blood samples arrived within 24 hours and $92 \%$ arrived within 48 hours of being mailed. No sample was damaged during the post and only 
two samples were so severely haemolysed as to prevent analysis.

DIFFERENCES IN SELECTED CHARACTERISTICS OF RESPONDERS AND NON-RESPONDERS

There were no differences in the overall mean levels when measured in 1970 of systolic or diastolic blood pressure, total cholesterol, height, weight or body mass index between those men in the random sample who completed both a questionnaire and provided a blood sample in 1995 ("compliers") and those who did not (table 2). There was also little change in the subsample of compliers between the overall mean values of systolic and diastolic blood pressure and total cholesterol in 1970 and in 1995.

\section{CHANGES WITHIN SUBJECTS BETWEEN 1970 AND} 1995

In contrast with the absence of significant changes in the overall mean values of systolic and diastolic blood pressure and of blood total cholesterol, the mean values in 1995 for quintiles based on 1970 values were substantially different from their mean values in 1970 (table $3)$. The ratio of the difference between the upper and lower mean quintiles in 1970 and that in 1995 was 3.5 for each of systolic blood pressure, diastolic blood pressure, and total cholesterol. Similarly, the inverse of the correlation coefficients between values in 1970 and those in 1995 were 3.8 for systolic blood pressure, and for diastolic blood pressure and 2.6 for total cholesterol. By contrast, there was much less regression dilution for height and weight during the 25 year period. The effect of illness on these estimates of regression dilution bias was examined by excluding the 59 men ( $24 \%$ of the participants with blood) who reported a prior heart attack, angina, stroke or any type of cancer other than skin cancer. Among the men with no such history, the inverse of the correlations between 1970 and 1995 were not materially changed: 3.1 for systolic blood pressure, and 2.3 for total cholesterol. After excluding the 58 (25\%) men receiving blood pressure lowering medication, the inverse of the correlation coefficient was 3.0 for systolic blood pressure and after excluding $42(18 \%)$ men taking lipid lowering medication, the inverse correlation coefficient was 2.6 for total cholesterol (table 4).

\section{Discussion}

This pilot study demonstrated the feasibility of conducting a re-survey of this study population after 25 years, with self completed questionnaires obtained from $73 \%$ of the sample. The study confirmed the value of using reminder letters, which boosted the response rate by $12 \%$ after the first reminder and by $6 \%$ after the second reminder letter.

The use of "recorded" delivery established that $96 \%$ of the addresses provided by this system were accurate and that three quarters of the non-responders received the letters and were unable or unwilling to reply. The response rate compares favourably with that of the initial survey in 1968 to 1970 when $77 \%$ responded, ranging from $58 \%$ in the lowest employment grade to $87 \%$ in the senior grades. ${ }^{11}$

It is not always appreciated that self administered questionnaires require a high rate of literacy and reading skills. For this reason, the number of open ended questions had been kept to a minimum as "closed" questions are easier to answer, merely requiring the respondent to recognise an item of information rather than recall it. Moreover, the cover letter indicated that respondents could obtain help to complete the questionnaire. Even so, some of the non-respondents, or a family member, indicated by telephone to the study office that they still felt unable to complete this 16 page questionnaire. Hence, in the main re-survey nonresponders after two mailings will be sent a single page questionnaire seeking basic information on medical history, smoking status, self rating of health, age, and employment grade at retirement in the civil service.

The quality of mailed whole blood samples has previously been shown to be adequate for many biochemical investigations (such as assays of blood lipids, fibrinogen and plasma cotinine, and isolation and analyses of DNA). ${ }^{17} 18$ General practices were extremely cooperative, with requests for information on blood pressure, height, and weight and a blood sample received from $84 \%$ of the study participants who had completed a questionnaire and were sent a blood collection kit. In view of the geographical distribution of the study participants throughout the UK, the collection of whole blood samples by post was a cost effective method of obtaining this information. Using first class post ensured that over $90 \%$ of the samples arrived within 48 hours (with only two of these samples so haemolysed as to be unusable).

The response rate in this study compared favourably with the response rate of $70-75 \%$ obtained by other UK surveys, such as the General Household Survey, which collected information on household income using interviewers. ${ }^{19}{ }^{20}$ Some epidemiological studies have not sought data on income routinely because of concerns about an adverse effect on the response rate. This study, however, indicated that the response rate was unaffected by asking about the sources of income and the average net weekly income after tax. By contrast, seeking information on cognitive function using an informant questionnaire and Short Blessed test administered by the GP did seem to have an adverse effect on the response rate for questionnaires and for blood samples. Consequently, assessment of cognitive function has been omitted from the main re-survey.

Obtaining accurate current addresses was a prerequisite for carrying out a re-survey of the study participants after 25 years. In 1968 to 1970 all of the men were in government employment in central London within about a two mile radius of Whitehall. ${ }^{7}$

Using information obtained from the NHSCR, the survivors living in the United Kingdom were found to be registered with a family doctor in 96 health authorities. For the 
pilot study, current addresses of study participants were obtained manually from three health authorities and found to be accurate for $96 \%$. But, although health authorities were extremely cooperative, this method of obtaining current addresses was very time consuming. Hence, computer software has been developed to obtain current addresses from the 96 health authorities for the main re-survey of the remaining 8400 survivors in the UK.

An important finding from the pilot study, which is discussed in detail elsewhere ${ }^{10}$ was the magnitude of within person change in blood pressure and cholesterol values. Regression dilution after about 26 years in the Whitehall study was greater than had been anticipated from previously reported studies with shorter intervals between re-measurements. This implies that, even after excluding those who had prior disease or taking relevant therapy, risk factors measured only in middle age may lead to substantial underestimates of the strength of associations with risk in long term follow up of prospective studies. This study reinforces the value of replicate measurements of exposures at periodic intervals during follow up in prospective studies to obtain reliable estimates of age specific mortality with risk factors.

Richard Peto, Mary Burton, Heather Hall, Steve Sutherland, Harry Hemingway, Martin Prince and Pamela Bell made helpful contributions to this study. This re-survey would not have been possible without the collaboration of the chief executives of the health authorities, the FH Computer Unit at Exeter, and the National Health Service Central Register.

Funding: the re-survey is supported by a project grant from the British Heart Foundation (PG 96075).

1 McMahon S, Peto R, Cutler J, et al. Blood pressure, stroke and coronary heart disease; prolonged differences in blood pressure; prospective observational studies corrected for pressure; prospective observational studies correcte
regression dilution bias. Lancet 1990;335:765-74.

2 Neaton JD, Wentworth D. Overall findings and differences by age for 316,099 white men. Multiple Risk factor Intervention Trial Research Group. Arch Intern Med 1992;152 $56-64$
3 Glynn RJ, Field TS, Rosner B, et al. Evidence for a positive linear relationship between blood pressure and mortality in linear relationship between blood pressure

4 Benfante R, Hwang LJ, Masaki K, et al. To what extent do cardiovascular risk factors measured in elderly men represent their mid-life values measured 25 years earlier? A preliminary report from the Honolulu Heart Study. Am $\mathcal{F} E p i$ demiol 1994;140:206-16.

5 WHO Technical Report 853. Epidemiology and prevention of cardiovascular diseases in elderly people. Report of a WHO Study Group. Geneva: WHO, 1995.

6 Marmot MG, Shipley MJ. Do socioeconomic differences in mortality persist after retirement? 25 year follow-up of civil servants from the first Whitehall study. BMF 1996;313: $1177-80$.

7 Gardner MJ, Heady JA. Some effects of within-person variability in epidemiological studies. F Chronic Dis 1973;26: 781-95

8 Davis CE, Rifkind BM, Brenner $\mathrm{H}$, et al. A single cholesterol measurement underestimates the risk of coronary heart disease: an empirical example from the Lipid Research Clinics Mortality Follow-up. $7 A M A$ 1990;264:3044-6.

9 Tornberg SA, Jakobsson KFS, Eklund GA. Stability and validity of a single serum cholesterol measurement in a validity of a single serum cholesterol measurement in a

10 Clarke R, Shipley M, Collins R, et al. Underestimation of risk associations due to regression dilution in long-term follow-up of prospective studies. Am $\mathcal{F}$ Epidemiol (in press).

11 Reid DD, Brett GZ, Hamilton PJ, et al. Cardiorespiratory disease and diabetes among middle-aged male civil servants: a study of screening and intervention. Lancet 1974;i:469-73.

12 Reid DD, Hamilton PJS, McCartney P, et al. Smoking and other risk factors for coronary heart disease. Lancet 1976;ii:979-84.

13 Richie K, Fuhrer R. A comparative study of the performance of screening tests for senile dementia using receiver operating characteristics analysis. F Clin Epidemiol 1992;45: 627-37.

14 Blessed G, Tomlinson BE, Roth $M$. The association between quantitative measures of dementia and of senile change in the cerebral gray matter of elderly subjects. $\mathrm{Br} \mathcal{F}$ Psychiatry 1968;149:698-709.

15 Shipley MJ, Pocock SJ, Marmot MG. Does plasma cholesterol concentration predict mortality from coronary heart disease in elderly people? 18-year follow-up in the Whitehall study. BMf 1991;303:98-2.

16 Davey-Smith G, Shipley MJ, Marmot M, et al. Plasma cholesterol and mortality: the Whitehall study. $\mathcal{F A M A} 1992$; 267:70-6.

17 Parish S, Peto R, Collins R, et al for the ISIS Collaborators. Cigarette smoking, tar yields and non-fatal myocardial infarction: 14000 cases and 32000 controls in the United Kingdom. BMf 1995;311:471-7.

18 Youngman LD, Lyon V, Collins R, et al. Problems with mailed blood in large scale epidemiological studies and methods of correction. FASEB $\mathcal{F}$ 1993;1:377.

19 Breeze E, Trevor G, Wilmot A. The general household survey 1989. London: HMSO, 1991.

20 Rowlands O, Singleton N, Maher J, et al. Living in Britain in the 1995 general household survey. London: HMSO, 1996. 